\title{
A Nonlinear Electro-Thermal Scalable Model for High Power RF LDMOS Transistors
}

\author{
John Wood, Fellow, IEEE, Peter H. Aaen, Member, IEEE, Daren Bridges, Member, IEEE, \\ Dan Lamey, Member, IEEE, Michael Guyonnet, Member, IEEE, Daniel S. Chan, Member, IEEE, and \\ Nelsy Monsauret
}

\begin{abstract}
A new nonlinear, charge-conservative, scalable, dynamic electro-thermal compact model for LDMOS RF power transistors is described in this paper. The transistor is characterized using pulsed I-V and S-parameter measurements, to ensure isothermal conditions. A new extrinsic network and extrinsic parameter extraction methodology is developed for high power RF LDMOS transistor modeling, using manifold de-embedding by electromagnetic simulation, and optimization of the extrinsic network parameter values over a broad frequency range. The intrinsic model comprises controlled charge and current sources that have been implemented using artificial neural networks (ANNs), designed to permit accurate extrapolation of the transistor's performance outside of the measured data domain. A thermal sub-circuit is coupled to the nonlinear model. Largesignal validation of this new model shows a very good agreement with measurements at $2.14 \mathrm{GHz}$.
\end{abstract}

Index Terms - field effect transistor, LDMOS, nonlinear, transistor model

\section{INTRODUCTION}

$\mathbf{L}$ ATERALLY-DIFFUSED MOS (LDMOS) FETs are used almost exclusively for high power transistors for wireless infrastructure or base-station applications. They provide an unmatchable combination of performance and cost, and are capable of delivering hundreds of watts of RF power.

This combination of high powers and high frequencies in $\mathrm{RF}$ and microwave power amplifiers brings together a unique set of challenges for the device modeling engineer. The power transistors themselves are physically large, and may be a significant fraction of a wavelength wide, even at microwave frequencies. The electrical behavior in this distributed environment must be captured in the model. The device will generate a lot of heat, and the thermal effects on the transistor's electrical behavior will also need to be characterized and included in the model. Further, power amplifiers for wireless communications systems are tightly specified in terms of their linearity performance, bandwidth, etc., placing a premium on the availability of accurate nonlinear transistor models.

In this paper we shall outline a new nonlinear scalable model for the LDMOS transistor, and its extraction and implementation. The transistor model architecture is shown in Fig. 1. The intrinsic part of the model, shown in the center of the figure, describes the nonlinear behavior of the transistor, using

J. Wood, P. H. Aaen, D. Bridges, D. Lamey, M. Guyonnet and D. Chan are with the RF Division, Freescale Semiconductor Inc., Tempe, AZ 85284 (e-mail: John.Wood@freescale.com)

N. Monsauret is with the RF Division, Freescale Semiconductor Inc., Toulouse France.

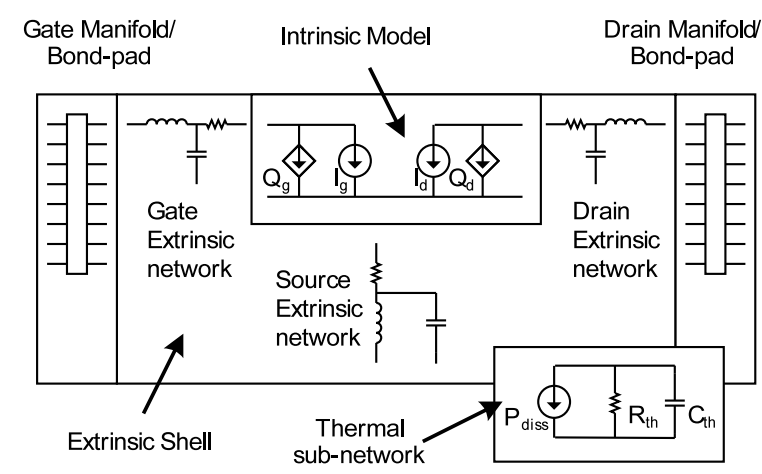

Fig. 1. Block representation of the transistor model architecture.

voltage-controlled current and charge sources: the model state functions or constitutive relations. This basic structure is no different in principle to those nonlinear models presented by [1]-[3]. A charge-conservative approach is adopted, as this is crucial to the accurate prediction of the low-level nonlinearities such as those described by intermodulation products, adjacent channel power, and so forth [4]. Included in this model are accurate, continuously-differentiable functions for the model currents and charges, and a self-consistent electro-thermal model coupled to these controlled sources.

These model functions are designed to compute, rapidly and accurately, values of the constitutive relations anywhere within the measured characterization plane. It is also necessary for these functions to be able to handle evaluations of the state functions outside of the measured data range; in other words, these functions must have predictable and controlled extrapolation properties. The reason for this is that in circuit simulation, as the simulation iterates towards the solution, the harmonic balance simulator (for example) may at some point require a solution for input voltages that lie outside the measurement space. Poor extrapolation behaviour can lead to non-convergence even if the final solution exists within the characterized region [5]. Here we present also a new formulation for approximating the state functions for a measurementbased model that permits well behaved and accurate extrapolation. The methodology is extended to include the thermal dependency of the state-functions.

The extrinsic network describes the electrical behaviour of the transistor layout; it accounts for the metallizations, substrate, and semiconductor that connect the intrinsic model to the outside world. How the electrical behaviour of this network changes with device size determines how the extrinsic 


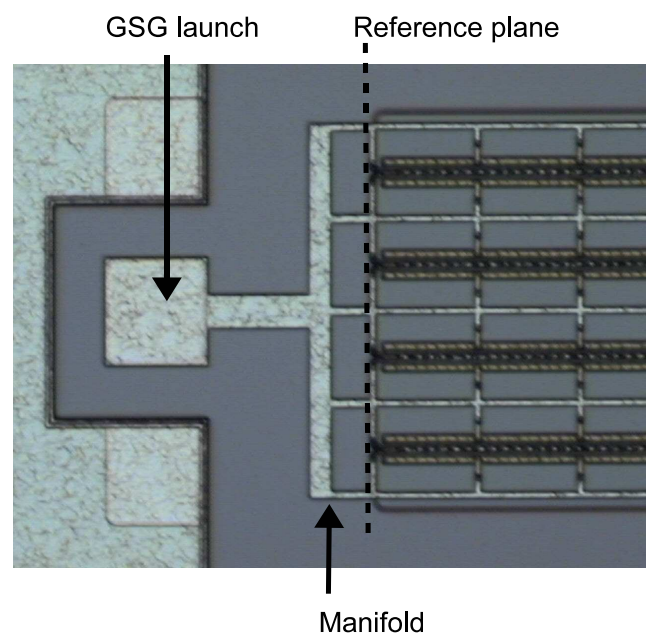

Fig. 2. Discrete LDMOS transistor used for device modeling; this structure is GSG probe-able, and the reference planes for the manifold are shown.

network component values scale. Scaling is a significant consideration: the RF power transistor may have a total gate width of over $100 \mathrm{~mm}$ : this is impossible to model in a traditional small-signal S-parameter environment - the RF powers are too high, and the device impedances are too small to permit measurements of sufficient accuracy for model extraction, if indeed the device is stable in a $50 \Omega$ environment. Instead, we measure and extract a model for a smaller device structure, and scale this model to the appropriate size.

\section{TRAnsistor CHARACTERIZATION}

The transistor characterization is carried out on-wafer. The test transistor has the same basic structure as the production device, but is much smaller: it has fewer gate fingers. The RF calibration to the Ground-Signal-Ground (GSG) probe tips is carried out using an impedance standard substrate. The current-voltage and S-parameter measurements are all made under pulsed conditions. A low pulse duty cycle is used to ensure that the data are captured isothermally. Using pulsed conditions also enables us to measure beyond the DC dissipation limit of the device, into realistic instantaneous voltage and current regimes. A dense pattern of the pulsed $\mathrm{I}-\mathrm{V}$ and S-parameter measurements is made over the gatedrain voltage space of the transistor, bounded by the maximum drain current, breakdown voltage, and the maximum allowable power dissipation.

\section{MAnifold Modeling \& De-Embedding}

Figure 2 shows the metallization in a typical test structure used for transistor model development. The problem is segmented into two areas of analysis, viz the GSG launches and the manifolds. The goal here is to remove the effects of the launch and manifolds, leaving only the response of the transistor including extrinsic elements. The electrical behaviour of the GSG launches is determined from measurements of transmission line structures, with care taken over the placement of the calibration reference plane. The GSG launches can then be modeled by error boxes placed at the

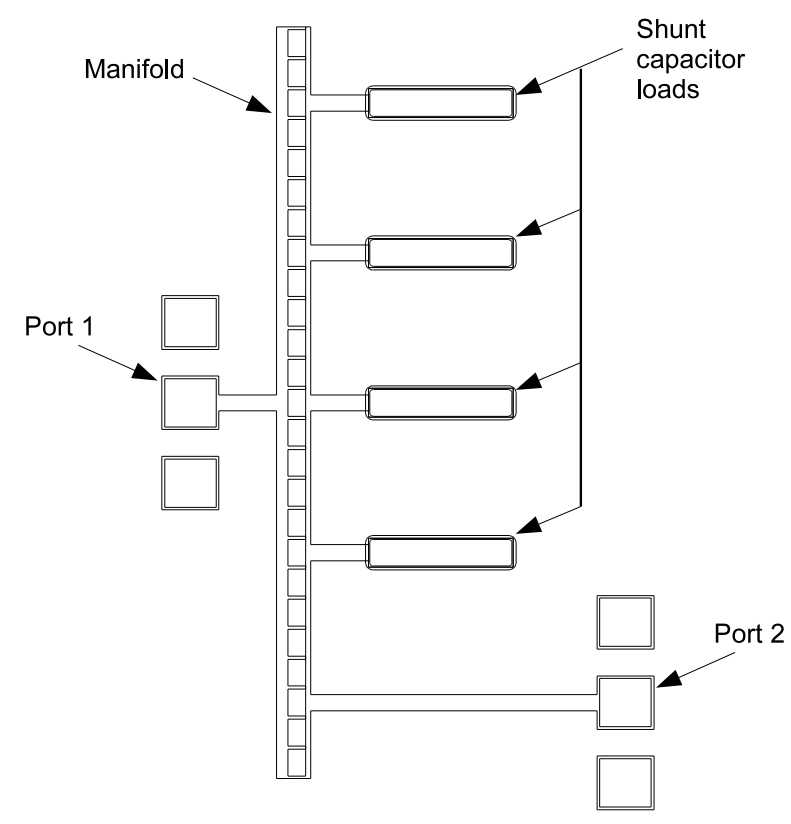

Fig. 3. Manifold test structure for electromagnetic simulation showing shunt capacitor loads.

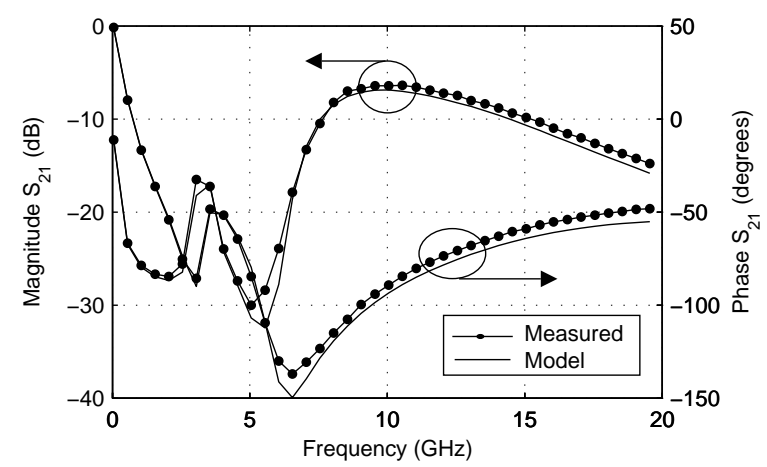

Fig. 4. Measured versus EM-modeled transmission characteristics for the structure shown in Fig. 3, indicating excellent broadband agreement.

measurement reference planes. The effects of the manifolds can then be determined, and the GSG measurements are then de-embedded to the reference plane shown in Fig. 2.

The behavior of the manifolds in turn is obtained through electromagnetic simulation, using Sonnet's $\mathrm{em}^{\mathrm{TM}}$. An accurate substrate definition is key to the manifold extraction. The substrate definition is found from careful analysis of measured S-parameters for transmission lines of various lengths and widths. By converting these S-parameters to the telegrapher's parameters for each line, the properties of each layer in the substrate and metal stack can be determined, and the appropriate metal model chosen. This is particularly challenging in LDMOS, because the silicon substrate is quite lossy.

The substrate definition is then used in the simulation of S-parameters for each of the manifold structures. For the results presented here, the wafer used for transistor modeling also included structures for determination of the simulation substrate definition. 
To validate the procedure, measured S-parameters of a loaded manifold were compared to a circuit simulator result for simulated manifolds plus measured loads. Large manifold 2port GSG structures were built, both with capacitive loads and unloaded. A typical structure is illustrated in Fig. 3. The loads were shunt capacitors placed periodically along the manifold width, with capacitance matching the effective capacitance of a typical LDMOS device under bias. S-parameters of a single such capacitor were also measured directly. Electromagnetic simulations of the manifolds were combined with the measured load S-parameters in a circuit simulator.

The two-port measurements of the loaded manifold structure, illustrated in Fig. 3, are in good agreement with simulations. The transmission through the loaded manifold structure is plotted against measured results in Fig. 4. Although not plotted, the remaining S-parameters are in similar agreement.

\section{Extrinsic Network Parameter Extraction}

The traditional extrinsic network for a MESFET comprises a series resistor-inductor network connected to each gate and drain, and source nodes of the intrinsic model - the Z-shell, followed by shunt capacitors from gate and drain to ground, and between gate and drain - the Y-shell [6].

For LDMOS transistors we have augmented this simple network with a resistor in parallel with the series gate \& drain inductance, to account for the frequency dependent behavior of the silicon substrate material in an empirical manner. Adding these shunt resistors enables an improved broadband fit of the return losses. Further, we have observed coupling effects between the gate and drain metallizations in these power transistors. The unit gate widths in these power transistors are quite significant, over $500 \mu \mathrm{m}$, and there is measurable mutual inductance between these long metal traces.

We use the Cold-FET method [6]-[8] as the basis for our extrinsic parameter extraction, using optimization over a broad frequency range. Our methodology is outlined below, and described in detail in [9]. Broadband S-parameters were measured under bias conditions of zero volts on gate and drain terminals of the transistor: this ensures the LDMOS transistor is biased below threshold, and is in the passive or 'Cold' condition. The manifolds were then de-embedded from these data by using their S-parameter blocks determined as described in Section III. The Cold-FET circuit for the intrinsic transistor is assumed to be a purely capacitive network. The extrinsic network and Cold-FET circuit are illustrated in schematic form in Fig. 5.

The extrinsic network parameter values were extracted from this de-embedded data using the following sequence of actions: first, the extrinsic network components were de-embedded to obtain intrinsic S-parameters; next, the Cold-FET intrinsic parameter values were calculated from these intrinsic S-parameters; then this intrinsic model was reembedded with the extrinsic network; and finally these model S-, Y-, and Z-parameters were compared to the measured values (after de-embedding of the manifolds). The extrinsic network parameters were adjusted until the measured and model data were suitably close, over the broadband frequency

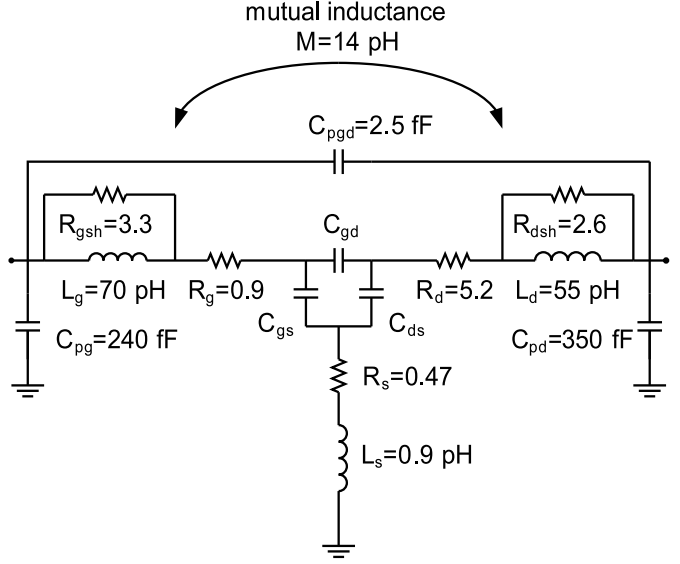

Fig. 5. The new extrinsic network with the Cold-FET intrinsic circuit also shown, for a transistor with a total gate periphery of $4.8 \mathrm{~mm}$.
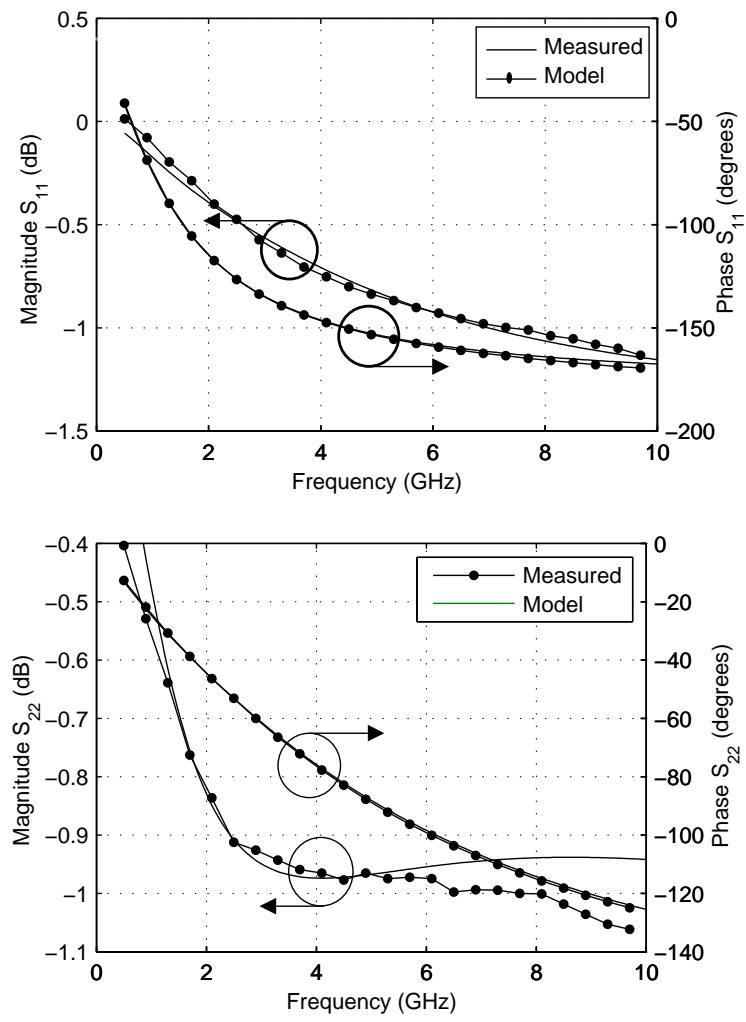

Fig. 6. Comparison between measured and modeled manifold-de-embedded S-parameters.

range. Also, we ensured that the resulting small-signal intrinsic component parameters were physically realistic, for example, the model resistances were non-negative. This procedure was performed in Agilent-EEsof ADS ${ }^{\mathrm{TM}}$, using the Sequencer component and the Tuning simulator. As a starting point, either nominal or zero values for the extrinsic network components can be used with equal success, indicating the robustness of the technique. The broadband S-parameters for the small-signal model and extrinsic network are compared to the measured and de-embedded S-parameters in Fig. 6, showing excellent agreement. 


\section{NONLinear InTRINSIC MOdel}

The manifold structure and the extrinsic network are deembedded to obtain S-parameter data at the intrinsic model reference planes [9]. After converting to Y-parameters, the LDMOS transistor model current and charge state functions can then be obtained by integration of the small-signal voltagedependent parameters, using the following equations [3].

$$
\begin{aligned}
I_{\mathrm{d}}\left(V_{\mathrm{gs}}, V_{\mathrm{ds}}\right) & =\int_{V_{\mathrm{gs} 0}}^{V_{\mathrm{gs}}} g_{\mathrm{m}}\left(v_{\mathrm{gs}}, V_{\mathrm{ds} 0}\right) d v_{\mathrm{gs}}+\int_{V_{\mathrm{ds} 0}}^{V_{\mathrm{ds}}} g_{\mathrm{ds}}\left(V_{\mathrm{gs}}, v_{\mathrm{ds}}\right) d v_{\mathrm{ds}} \\
& +I_{\mathrm{d}}\left(V_{\mathrm{gs} 0}, V_{\mathrm{ds} 0}\right) \\
Q_{\mathrm{g}}\left(V_{\mathrm{gs}}, V_{\mathrm{ds}}\right) & =\int_{V_{\mathrm{gs} 0}}^{V_{\mathrm{gs}}}\left[C_{\mathrm{gs}}\left(v_{\mathrm{gs}}, V_{\mathrm{ds} 0}\right)+C_{\mathrm{gd}}\left(v_{\mathrm{gs}}, V_{\mathrm{ds} 0}\right)\right] d v_{\mathrm{gs}} \\
& -\int_{V_{\mathrm{ds} 0}}^{V_{\mathrm{ds}}} C_{\mathrm{gd}}\left(V_{\mathrm{gs}}, v_{\mathrm{ds}}\right) d v_{\mathrm{ds}}+Q_{\mathrm{g}}\left(V_{\mathrm{gs} 0}, V_{\mathrm{ds} 0}\right) \\
Q_{\mathrm{d}}\left(V_{\mathrm{gs}}, V_{\mathrm{ds}}\right) & =\int_{V_{\mathrm{gs} 0}}^{V_{\mathrm{gs}}}\left[C_{\mathrm{m}}\left(v_{\mathrm{gs}}, V_{\mathrm{ds} 0}\right)-C_{\mathrm{gd}}\left(v_{\mathrm{gs}}, V_{\mathrm{ds} 0}\right)\right] d v_{\mathrm{gs}} \\
& +\int_{V_{\mathrm{ds} 0}}^{V_{\mathrm{ds}}}\left[C_{\mathrm{ds}}\left(V_{\mathrm{gs}}, v_{\mathrm{ds}}\right)+C_{\mathrm{gd}}\left(V_{\mathrm{gs}}, v_{\mathrm{ds}}\right)\right] d v_{\mathrm{ds}} \\
& +Q_{\mathrm{d}}\left(V_{\mathrm{gs} 0}, V_{\mathrm{ds} 0}\right)
\end{aligned}
$$

The integrations are carried out starting from the quiescent bias point, denoted by $\left(V_{\mathrm{gs} 0}, V_{\mathrm{ds} 0}\right)$. Typically for LDMOS power transistors this corresponds to Class $\mathrm{AB}$ quiescent bias conditions: $V_{d d}=28 \mathrm{~V}, I_{d}=6 \mathrm{~mA} / \mathrm{mm}$, corresponding to $V_{g s} \approx 2.7 \mathrm{~V}$. Also for LDMOS, The gate current source is set to zero.

\section{A. Drain Current Model}

The drain current expression given by (1) is a 'high frequency' drain current, and using this for the model at RF overcomes some of the dispersion issues that are present in some FET technologies, such as trap-related dispersion, for example. Even though LDMOS does not suffer from significant trap-induced dispersion, we use the high frequency drain current to derive the model so that the model generation process is a generic one that can be applied to different FET technologies.

In our nonlinear model implementation, we can choose from a number of possible drain current functions, all of which are analytical expressions that result in a smooth and differentiable relationship, enabling accurate prediction of high order nonlinearities, and better interpolation, or, more strictly, function approximation, between the measured data points.

One example of a drain current function approximation that we can use is the analytical expression due to Fager et al. [10] to fit the high frequency drain current data [11]. This expression has an improved fit, compared with earlier
FET models, to the drain current data in the near-threshold region, which is typically where the LDMOS power transistor is biased for power amplifier applications.

The focus of this work is the use of artificial neural networks (ANNs) to approximate the drain current data. This approach shows similar levels of accuracy as methods of function approximation using elementary functions with fitting parameters [3]. The function approximation using ANNs is described in more detail in Section VI-A, in the context of controlled extrapolation. In addition to this ANN model, which is used to fit the data in the active on-state and threshold regions, we add functions to mimic the drain current behavior in the on- and off-state breakdown regions of the FET. These breakdown currents are modeled using diode-like expressions, as used in the Motorola Electro-Thermal (MET) model [12]. It is essential to include these effects in the model for largesignal applications.

\section{B. Charge Model}

One of the main objectives of this new nonlinear model development was to improve the charge model description over that of the existing MET model. The integral formulation for determining the charges described by eqns. (2) and (3) ensures a conservative charge formulation, which is essential for accurate prediction of low-level phase nonlinearity, and for convergence in time-domain simulations [3], [13]. A chargeconservative model formulation, approximated by a smooth and differentiable function, and capable of including temperature dependence, was seen as an essential goal for this model.

The conservative gate and drain charges, $Q_{g}$ and $Q_{d}$ of (2) and (3) were obtained through a Root model extraction from the measured intrinsic data, using the commercial AgilentEEsof IC-CAPTM function. In our model extraction, this produces tables of the charge data indexed by the intrinsic gate and drain voltages. These data are then approximated using artificial neural networks (ANNs), resulting in smooth and infinitely differentiable two-dimensional charge functions that are used directly in the model in Fig. 1. It is also possible to obtain the neural network charge functions by fitting an adjoint neural net to the small-signal capacitances, avoiding the direct integration step in (2) and (3) [14].

\section{EXTRAPOLATION CONSIDERATIONS FOR LARGE-SIGNAL DEVICE MODELING}

Extrapolation is a major concern for high power RF transistor modeling. The high current densities associated with power transistors, and the limited power and current capabilities of the measurement instrumentation often mean that the region of the device output characteristics that can be accessed and measured is much smaller than the region that is covered in RF operation of the transistor. This is illustrated in Fig. 7, which shows a dynamic load-line under RF drive superimposed on the measured DC I-V characteristics. While the dynamic loadline includes displacement currents, it can be seen from this illustration that the loadline trajectory can have a significant excursion into the unmeasured region. Hence, the extrapolation 


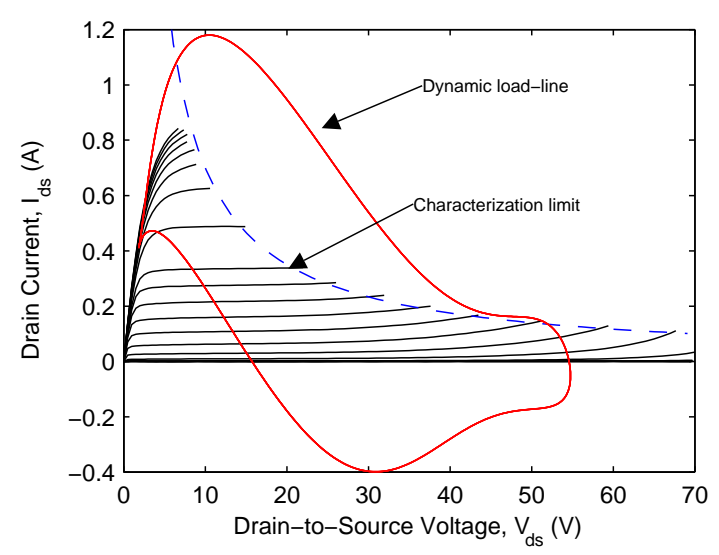

Fig. 7. A load-line for a transistor operating under mismatched conditions superimposed upon the drain current (under pulsed operation).

characteristics of the model state functions needs to be well behaved and accurate.

Extrapolation is the ability of the function approximation to return a value for input data that is outside the original training or characterization region. Generally, this statement is taken to mean that the extrapolated value returned by the function approximation is sufficiently close to the actual or true value of the data outside the training region. While this is one requirement of successful extrapolation, there is a further consideration for the simulation of large-signal models. The circuit simulator uses an optimization algorithm to determine the circuit voltages and currents, and for successful convergence there must be no local minima in the solution domain. The model nonlinear functions must therefore be extrapolated in such a way as to avoid these non-physical local minima: the extrapolation of the function outside the characterization data set should be able to redirect the simulator into the characterization domain.

In Fig. 8 we plot the measured characterization data, region I, and the regions of extrapolation corresponding to accurate function approximation, denoted by region II, and to redirection of the function, denoted by region III. In region I, the characterized region, the model used must approximate the measured data accurately, to within some acceptable level of error. In region II, accurate extrapolation is required since the loadline of the transistor may pass through this region. In region III, the breakdown region, provided that the form of the breakdown currents is correctly modeled, the main emphasis is to ensure robust convergence of the simulator; fidelity to the actual drain current is less important.

\section{A. Artificial Neural Networks for Function Approximation and Extrapolation}

The universal approximation theorem was a key factor in establishing ANNs as a useful approximation method [15]. This theorem states that a network with a single hidden layer of neurons is capable of approximating any given function, with any degree of accuracy, provided that there are sufficient neurons, and that their activation functions are non-constant,

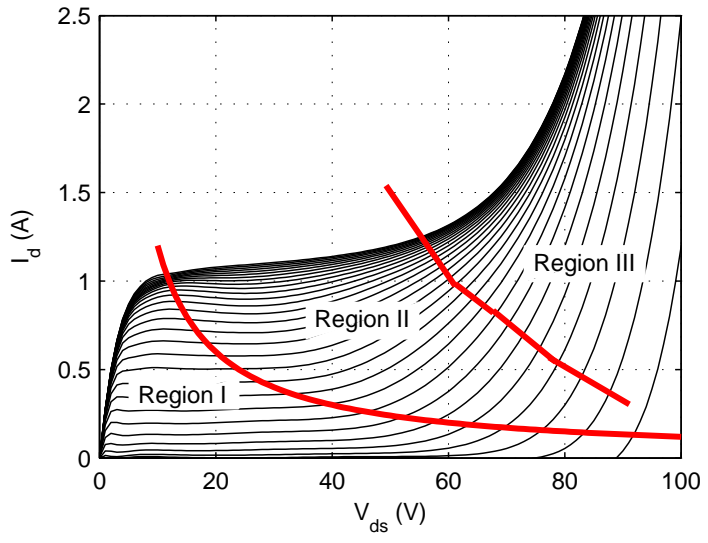

Fig. 8. An illustration of the various regions of the drain current. The measured characterization data is indicated by region I, while regions II \& III represent the extrapolation and breakdown regions.

bounded and monotonically increasing [16], [17]. A singlehidden-layer neural network function is expressed as

$$
\mathbf{y}=\sum_{i=1}^{n} a_{i} \mathrm{f}\left(\sum_{j=1}^{m} w_{j i} \mathbf{x}_{j}+b_{i}\right)+b_{o}
$$

where $\mathbf{y}$ is the data that is to be approximated, $\mathbf{x}$ is the input data, $a, b, w$ are the adjustable parameters of the function, $n$ is the number of neurons in the single hidden layer, and $m$ is the number of inputs - in our case this would be two: the gate and drain voltage values. The nonlinear activation function $\mathbf{f}$ of the neuron is typically a hyperbolic tangent function, and this is used in our model.

A known problem with a single hidden layer neural network is that the neurons tend to interact with each other globally: modifying the weights to make the approximation better at one point in the data will degrade it at another: the 'waterbed' effect. This global interaction can be overcome through the use of two hidden layers. Neurons in the first layer tend to partition the space into sub-domains and approximate the function locally. The second layer fits more global features. That is, the outputs of the first layer tend to operate only on localized areas of the function range and output zero elsewhere. The second layer combines these localized regions [16], [18].

We use a two-hidden-layer neural network for good approximation and extrapolation of the state function data. Further, a number of other techniques are used in the training of the neural network to improve the generality of the function approximation. One technique is that of regularization, where the performance function of the approximation is modified from a simple mean square error estimation of the function approximation. Often, this performance function is modified to include the mean of the sum of the squares of the weights and biases of the network. Bayesian regularization assumes that the weights and biases are random variables with given distributions, and the regularization is performed using statistical techniques. Another technique is that of 'early-stopping' and cross-validation, wherein the training data set is divided into several different subsets that can be used for training 
and validation of the neural network function approximation. The network is trained on one set of data, and the function approximation checked on another set of data: once the error in the approximation of the validation data set begins to increase, the training is terminated [19].

We use the Mathworks Matlab ${ }^{\mathrm{TM}}$ Neural Network Toolbox as our basic tool for training the artificial neural networks. We normalize the input and output data to ensure that the nonlinear neural network functions operate over their optimal range, and to prevent ill-conditioning of the optimization process. The training also employs a combination of the above methods to obtain high accuracy of approximation, and good generalization of the neural network. Using these techniques, the network output can represent the data accurately within the training region, and be well-behaved outside of the training region, as will be demonstrated later.

We have implemented a generic drain current expression using a combination of a neural-network and a breakdown model in the following manner,

$$
I_{d s}\left(V_{d s}, V_{g s}, T\right)=A N N\left(V_{d s}, V_{g s}, T\right) \times V_{b r}\left(V_{d s}, V_{g s}, T\right)
$$

where $T$ is the temperature, and $A N N$ is a neural network capable of an accurate approximation of the data over the measured characterization region. The breakdown model for $V_{b r}$ is of the following general exponential form

$$
V_{b r} \approx\left(1+k \mathrm{e}^{V_{b r k}}\right)
$$

where $k$ is a constant and $V_{b r k}$ is a temperature dependent breakdown voltage parameter. In fact, the actual breakdown model has more parameters than this to ensure good global fit; details of this breakdown model can be found in the documentation for the MET model [12].

This drain current formulation is very convenient to implement within a circuit simulator and the derivatives of $I_{d s}$ can be readily computed as it is the product of two differentiable functions. The parameters of the breakdown model are set so that the function has no influence on the neural network for the drain current within the characterized region and minimal influence within the extrapolation region. Extrapolation is governed the two layer feed-forward neural network and the breakdown model.

The extrapolation characteristics of the two-layer neural network are demonstrated using a set of drain current data obtained from measurement to train the network. The measurement system was limited to points with less than 15 watts dissipation: this defines the boundary between regions I \& II in Fig. 8. The neural network was trained only on data from this region. The neural network is then then used to predict the drain current in regions II \& III, and the results are plotted in Fig. 9, where it can be seen that the neural network predicts the drain current accurately within the training data range, and is well behaved in the extrapolation regions. The drain current plotted as a surface in Fig. 10, shows a smooth surface without local minima, and again the extrapolation is well behaved. Typically, we find that using two hidden layers, each containing between five and fifteen neurons, are sufficient for this level of accuracy and extrapolation performance.

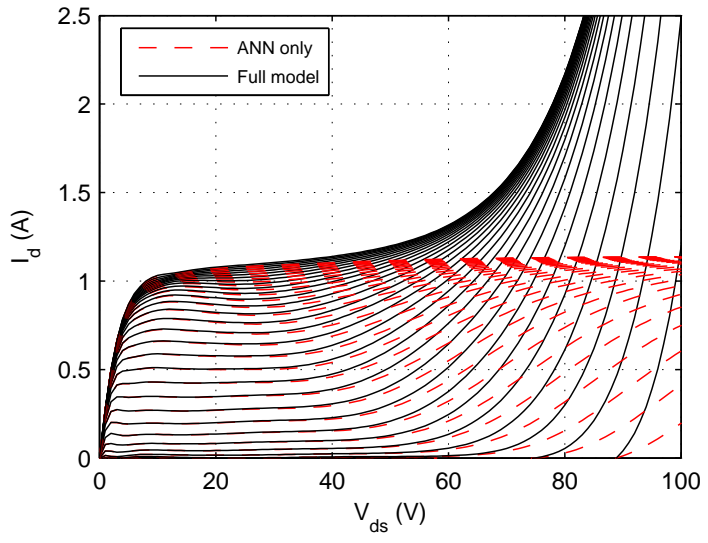

Fig. 9. A plot of the full drain current model including the breakdown model compared with the drain current as predicted by the neural network.

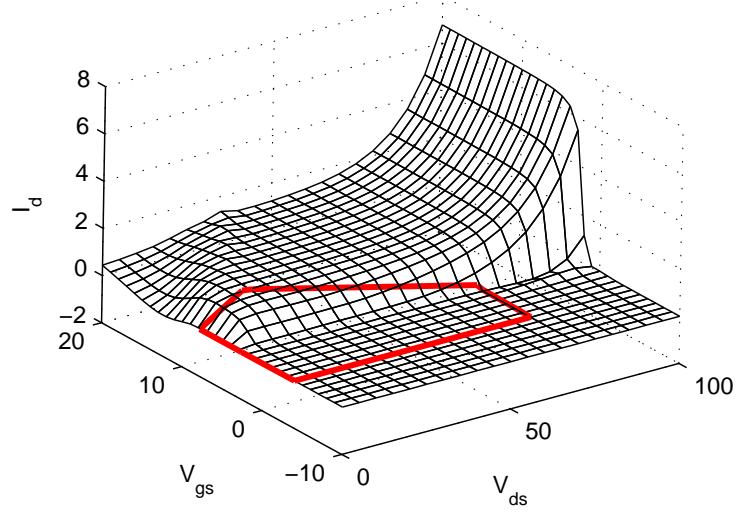

Fig. 10. A surface plot of the drain current as predicted by the full drain current model. The thick line indicates the range of voltages over which the drain current was measured.

\section{Model IMPLEMENTATION AND Verification}

The model has been implemented in ADS using the AgilentEEsof MINT ${ }^{\mathrm{TM}}$ modeling interface. A modular approach has been adopted allowing us to choose from and compare a variety of model implementations for the current and charge models: table-based models, MET expressions, neural networks, and the new model descriptions. This new model has been compared against the measured data, and existing MET and Root models extracted from the same data.

A $4.8-\mathrm{mm}$ gate periphery LDMOS transistor was characterized using pulsed I-V and S-parameter measurements, and these data were used to train the neural networks used to model this device. The measurement region was defined by several factors: a maximum permissible power dissipation for the transistor, a maximum permissible drain current, and a gatedrain breakdown voltage which has limits on the derivatives of $G_{d s}$ to prevent the measurement procedure from taking the transistor into on- or off-state breakdown regions where the possibility of permanent damage to the transistor occurs. The measurement procedure was repeated at three different chuck temperatures: 25,75 , and $125^{\circ} \mathrm{C}$. Approximately 1200 measurement points were captured at each temperature. 


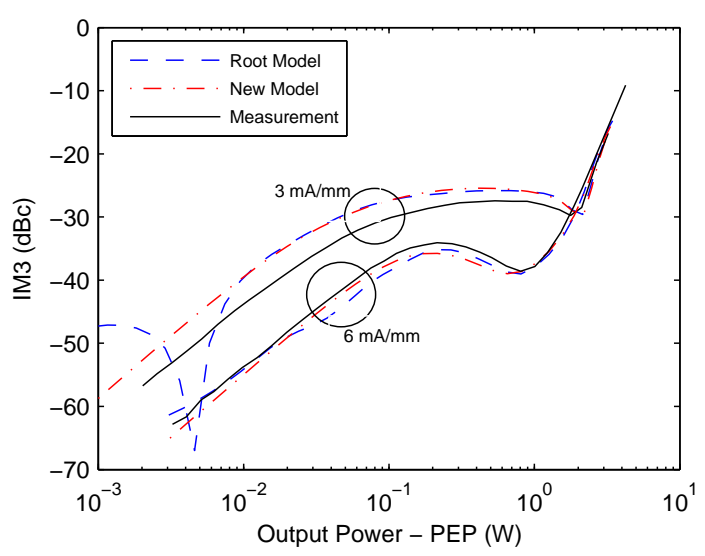

Fig. 11. Measured and simulated results of the intermodulation distortion products for $f_{o}=2.14 \mathrm{GHz}$ with a tone spacing of $100 \mathrm{kHz}$, as a function of the input power, at the intrinsic reference plane, for biases of 3 and 6 $\mathrm{mA} / \mathrm{mm}$ comparing MET and Root models to the new model.

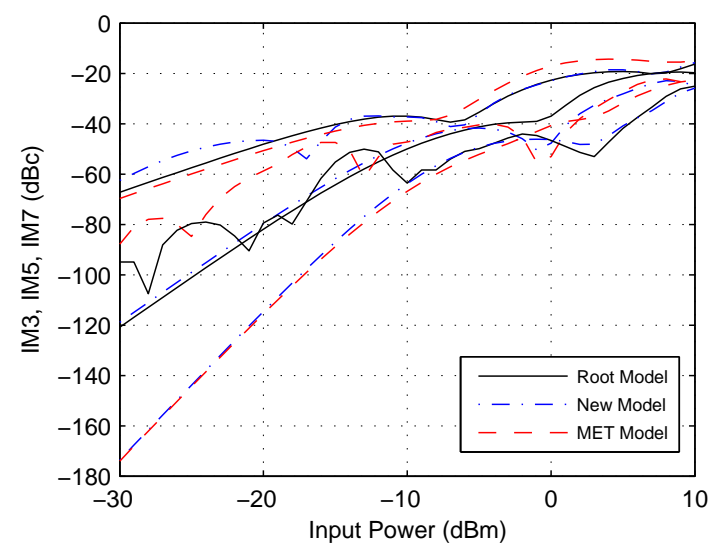

Fig. 12. Simulation results of the intermodulation distortion products for $f_{o}=2.14 \mathrm{GHz}$ with a tone spacing of $100 \mathrm{kHz}$, as a function of the input power, at the intrinsic reference plane, comparing MET and Root models to the new model.

The drain current model, presented earlier in Fig. 9, has very accurate approximation by the neural network within the measurement region, and reliable extrapolation beyond the measurement region boundary, giving confidence in the simulated drain current performance of this model.

The new model also displays excellent fidelity to the nonlinear device behaviour at RF frequencies. This is shown in Fig. 11, where we show that the new model predicts accurately the measured IM3 over a wide range of input drive. Also shown is the prediction using a table model: while this is accurate at high powers, at lower powers the interpolation can cause errors when the signal level is small. The new model also exhibits the correct asymptotic behavior of the intermodulation products at low drive powers. This is true also for higher order IM products as shown in Fig. 12, in contrast to table- or databased models, which are often limited by the order of the spline interpolation.

The approximation and extrapolation of the charge state functions are also well modeled by the neural networks. In Fig. 13 we show the gate charge as a function of gate and drain

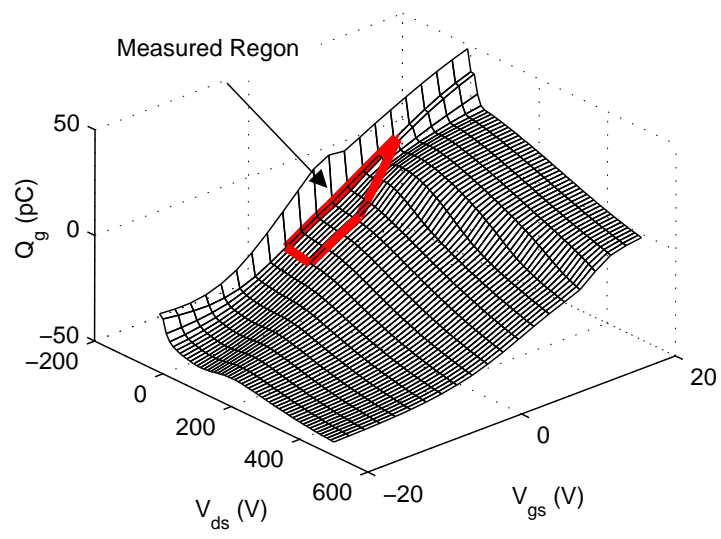

Fig. 13. A plot of $Q_{g}$ vs. $V_{d s}$ and $V_{g s}$. Outside the measured region indicated by the thick line, the charge surface predicted by the neural network is smooth and very well behaved, even at extremely high voltages which would never be experienced in practice, but may be used by the simulator during convergence.

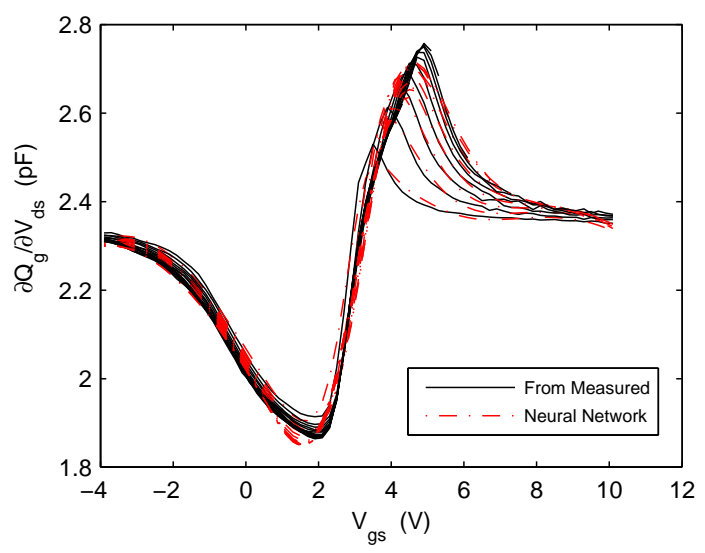

Fig. 14. Derivatives of the gate charge for various drain voltages plotted as a function of the gate voltage, $V_{g s}$.

voltages. The surface is well-behaved with no local minima or sharp gradients, and the extrapolation is smooth and wellbehaved. The measured region used during the neural network training process is also indicated on this figure. The charges exhibit only small temperature dependence, and the model also extrapolates well as a function of temperature. Similar results are found for the drain charge surface. As an illustration of the robust extrapolation property of this neural network formulation, we show the model gate charge function over an extreme range of drain voltage. The function is smooth, well-behaved, and convergent.

The derivatives predicted by the neural networks fit the numerical derivatives of the charge very well. In Fig. 14, the derivatives of the gate charge, as a function of gate voltage, for various drain voltages are plotted.

\section{Electro-Thermal Model}

Power transistors dissipate a lot of heat, and so it is necessary to include temperature dependence into the model parameters. Further, the information signals used in modern wireless communications have large peak-to-average ratios of 


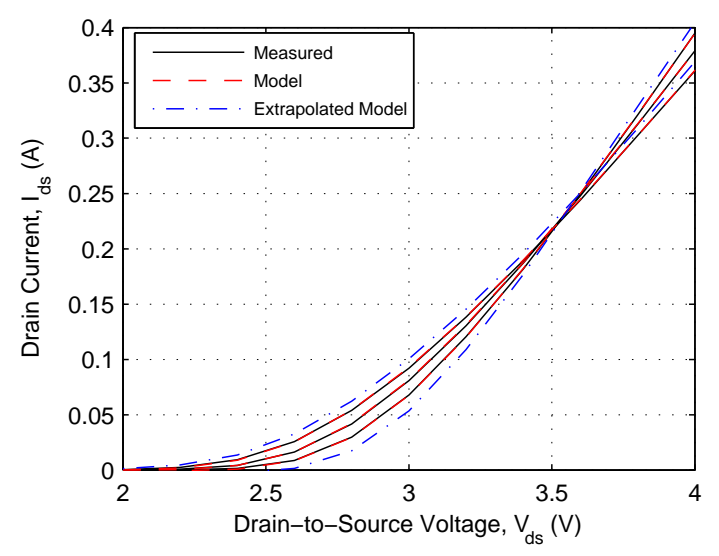

Fig. 15. The modeled and measured drain current is plotted at 25, 75, and $125^{\circ} \mathrm{C}$, as a function of the applied gate voltage. The drain voltage is 28 $\mathrm{V}$. In addition the model drain current is plotted at -25 and $150^{\circ} \mathrm{C}$ outside the measured range. This demonstrates the accurate prediction of the zerotemperature coefficient (ZTC) point.

the signal voltage or power: therefore, the dynamical response of the transistor's thermal behavior to these inputs must also be captured in the model. An accurate dynamical electro-thermal model is required.

A simple way of including the thermal effects on the output current is to use a de-rating function on the drain current expression [3], [11], [20].

The thermal variation of the threshold voltage acts in the opposite sense to the drain current de-rating, producing a point in the drain current-gate voltage relationship that is independent of temperature: the zero-temperature coefficient or ZTC point [21]. This threshold voltage variation is accommodated by an additional parameter in the drain current model, as in the MET model [12].

In addition to this accurate isothermal representation, the neural network model also returns accurate predictions of the drain current over temperature. In Fig. 15 we show the drain current dependence on the gate voltage in the region close to threshold, where competing thermal effects on the drain current exist, resulting in an observed point of thermallyindependent drain current: the 'zero temperature coefficient' current. The neural network predicts the nonlinear drain current behavior and its thermal dependences accurately, with the modeled and measured data being virtually indistinguishable. The neural network also predicts well behaved currents at temperatures outside the measured range, maintaining the ZTC point as expected.

\section{VAlidation OF THE MODEL}

The correct prediction of large-signal performance by the nonlinear model is also crucial. In Fig. 16 the drive-up characteristics of the model are compared with measurement at two different biases, showing very good agreement, even in compression. In Fig. 17 we show the measured transducer gain contours for a $4.8 \mathrm{~mm}$ gate-width LDMOS power transistor operating in Class-AB bias, taken at $2.14 \mathrm{GHz}$. This device is of identical layout to the device used for the model extraction. The model matches the contours and peak gain point with

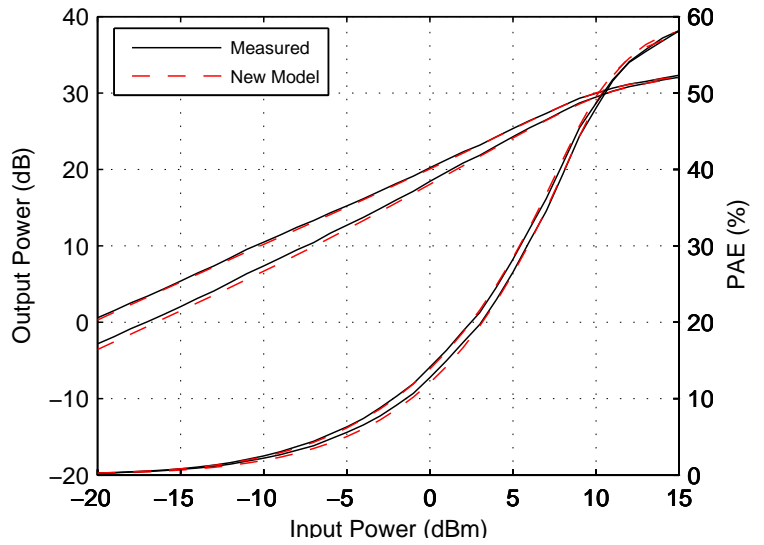

Fig. 16. Measured and modeled output power versus input power for bias currents equal to 6 and $9 \mathrm{~mA} / \mathrm{mm}$.

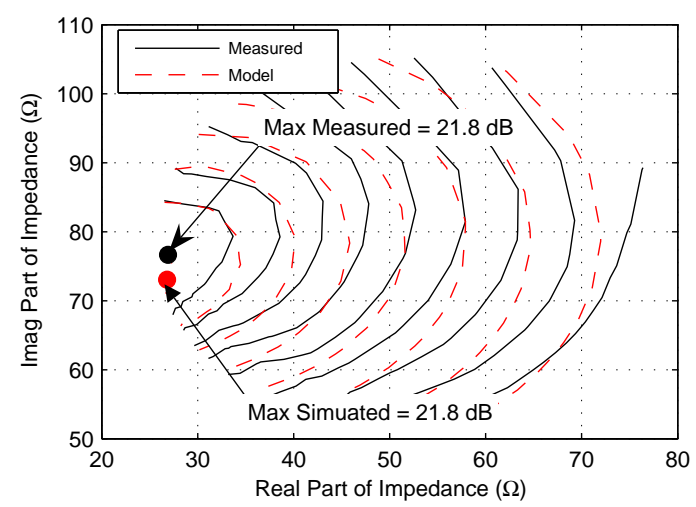

Fig. 17. Comparison between measured and modeled transducer gain loadpull contours at $\mathrm{P}_{1 d B}$. The contours are spaced in steps of $0.3 \mathrm{~dB}$.

TABLE I

COMPARISON OF THE ROOT AND NEW MODEL (TIME IN SECONDS).

\begin{tabular}{c||cccccc}
\hline & DC-IV & Envelope & HB 1-tone & LSSP & SP & Trans. \\
\hline Root & 1.97 & 67.90 & 6.19 & 3.73 & 1.39 & 24.73 \\
MET & 1.44 & 28.30 & 3.41 & 3.67 & 1.05 & 18.63 \\
This Model & 1.36 & 58.59 & 6.86 & 3.38 & 0.97 & 19.88 \\
\hline
\end{tabular}

excellent accuracy over the range of output powers and load impedances. The measured and predicted power-added efficiency load-pull contours for various output powers are shown in Fig. 18. This is a stringent test of a model: the large-signal, high-frequency nonlinear behavior and the DC conditions must be predicted accurately at the same time, and to do this over a range of load conditions and output powers indicates that the model is accurate, tracking the measured data very closely. This new model also executes in the simulator with no speed penalty, when compared with the industry-standard MET and Root models, as indicated in Table I. It should be noted that we have not yet optimized the new model for speed.

\section{Scaling Considerations}

The scaling behavior of the complete transistor model is heavily dependent on the scaling of the extrinsic network. Therefore, the scaling performance of the extrinsic network 


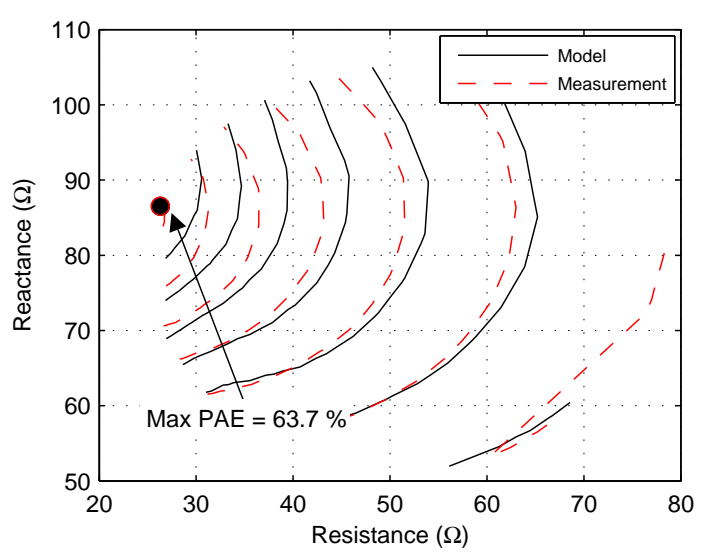

Fig. 18. Load-pull contours of power-added efficiency at $\mathrm{P}_{1 d B}$, comparing measured data and the new model. The contours are spaced in steps of $4 \%$.

parameters is a good test of how well this electrical behavior is modeled over a range of transistor gate peripheries or widths. For RF power transistors, the unit gate width is generally maintained constant for a given application, and scaling of the device is simply by the number of gate fingers. However, the unit gate width will be different for different applications. For example, the driver transistors in a multistage IC power amplifier will generally be much shorter than the power transistor gate widths; and the unit gate width will be larger for lower frequency applications, from considerations of overall die size. Moreover, from a modeling perspective, it is most useful to have one model kernel that can be used for all circuit and frequency applications, hence the ability to scale accurately with transistor gate periphery is extremely beneficial.

In Figs. 19-21 we illustrate the scaling behavior of the extrinsic resistance and inductance parameters. Four devices were chosen, having 4, 8, 16 and 32 gate fingers and a constant unit gate-width of $600 \mu \mathrm{m}$, yielding total gate peripheries of $2.4,4.8,9.6$ and $19.2 \mathrm{~mm}$, respectively. These devices were measured under Cold-FET conditions as outlined in Section IV, and the extrinsic parameters were extracted: the series gate and drain inductances; the gate, drain and source resistances; and the shunt gate and drain resistances. These extrinsic network parameters are seen to scale with total gate width in either a linear or inverse linear manner. The only outlier is the gate inductance of the 19.2-mm device: this is only one sample, and the higher than anticipated inductance may be a result of feeding a relatively wide device from a single point. Such simple and direct scaling indicates that the extrinsic network captures the electrical characteristics of the device layout very successfully. The extracted extrinsic capacitances are relatively independent of the gate periphery, over this range.

Further, as indicated in Fig. 5, we have identified a mutual inductance as a component of the extrinsic network, modeling the inductive coupling between the long fingers that are characteristic of power transistors. In Fig. 22 the extracted mutual inductance is shown as a function of the inverse of the total gate periphery for transistors with a unit gate width of

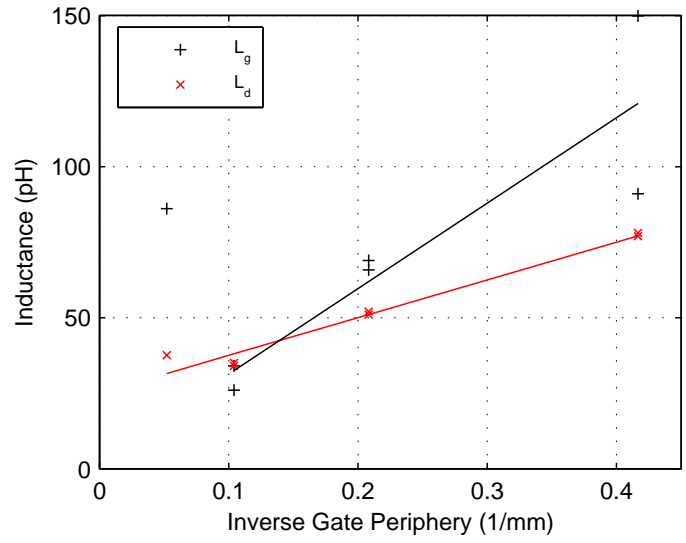

Fig. 19. Extrinsic series gate and drain inductance variation with the inverse of total gate periphery.

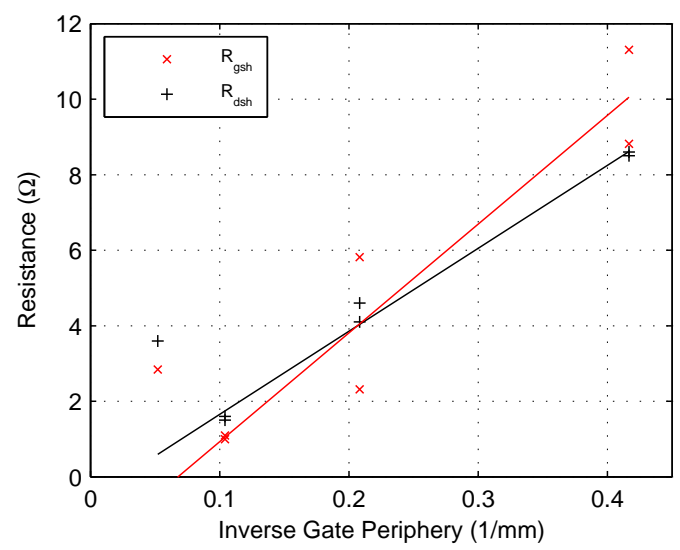

Fig. 20. Extrinsic gate and drain shunt resistance variation with the inverse of total gate periphery.

$600 \mu \mathrm{m}$. The mutual inductance is seen to scale inversely with gate width, or, alternatively, inversely with the number of gate fingers for a constant unit gate width.

\section{CONCLusion}

We have described the architecture, extraction and implementation of a new, nonlinear, charge-conservative, dynamic electro-thermal compact transistor model for RF power LDMOS FETs. This model includes a new extrinsic network for RF power LDMOS transistors, that is designed to accommodate features attributable to the lossy silicon substrate, and to the layout of the power transistor. The parameter extraction procedure takes advantage of sequencer and tuning capabilities of the circuit simulator to identify the network parameter values over a broad frequency range. The new network was observed to scale with gate width effectively and simply. The intrinsic part of the model uses voltage-controlled current and charge sources, these later being defined in a correct chargeconservative manner. These sources are implemented using two-hidden-layer artificial neural nets, and are shown to be accurate and to extrapolate well. The new Freescale ElectroThermal LDMOS FET (FET ${ }^{2}$ ) model is found to be more 


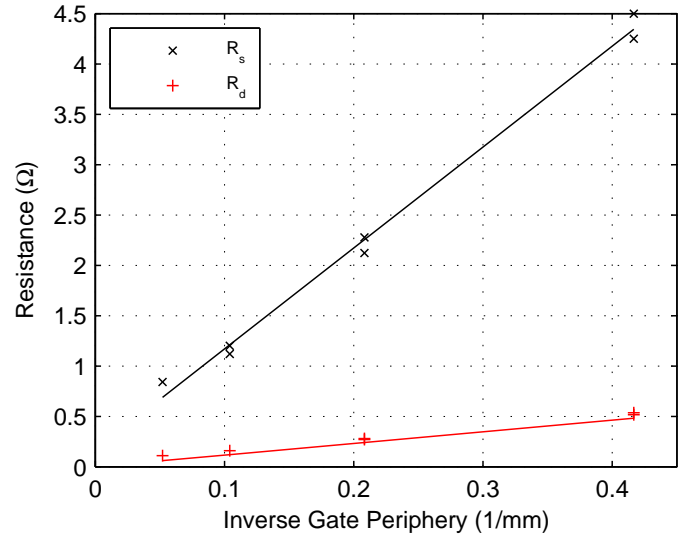

Fig. 21. Extrinsic drain and source resistance variation with the inverse of total gate periphery.

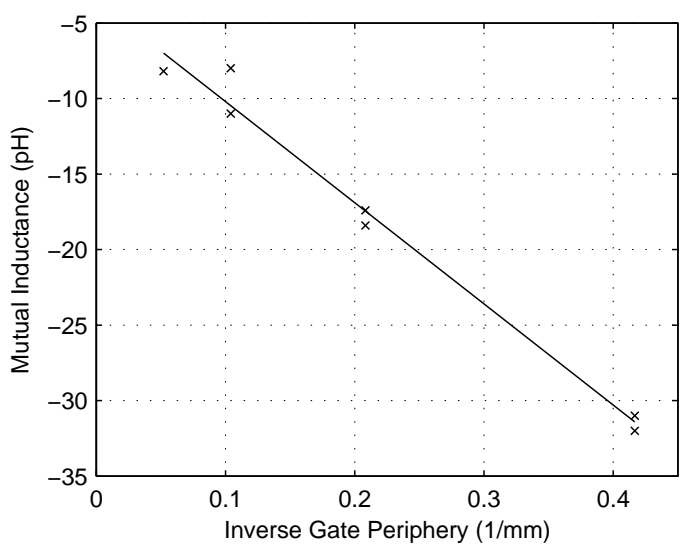

Fig. 22. Mutual inductance variation with the inverse of the total gate periphery.

accurate than both the industry-standard Motorola ElectroThermal (MET) model for power FETs and the isothermal Root model, which is another charge-conservative formulation. The new model is also at least as fast in simulation of typical power amplifier design applications, saving valuable design time.

\section{ACKNOWLEDGMENTS}

Thanks are due to the management staff of RF Division, Freescale, in particular Jaime Plá, for encouraging and supporting the development of this new nonlinear model.

\section{REFERENCES}

[1] D. E. Root, "Measurement-based mathematical active device modeling for high frequency circuit simulation," IEICE Trans. Electronics, vol. E82-C, no. 6, pp. 924-936, June 1999.

[2] P. Jansen, D. Schreurs, W. de Raedt, B. Nauwelaers, and M. van Rossum, "Consistent small-signal and large-signal extraction techniques for heterojunction FETs," IEEE Trans. Microwave Theory Tech., vol. 43, no. 1, pp. 87-93, Jan. 1995.

[3] P. H. Aaen, J. A. Plá, and J. Wood, Modeling and Characterization of RF and Microwave Power FETs. Cambridge, UK: Cambridge University Press, 2007.

[4] J. Staudinger, M. C. de Baca, and R. Vaitkus, "An examination of several large-signal capacitance models to predict GaAs HEMT linear power amplifier performance," in in Proc. IEEE Radio and Wireless Conf. (RAWCON), Colorado Springs, CO, Aug. 1998, pp. 343-346.

[5] J. Xu, M. C. E. Yagoub, R. Ding, and Q.-J. Zhang, "Robust neural based microwave modeling and design using advanced model extrapolation," in IEEE MTT-S Int. Microwave Symp. Dig., Fort Worth, TX, June 2004, pp. 1459-1552.

[6] G. Dambrine, A. Cappy, F. Heliodore, and E. Playez, "A new method for determining the FET small-signal equivalent circuit," IEEE Trans. Microwave Theory Tech., vol. 36, no. 7, pp. 1151-59, July 1988.

[7] D. Lovelace, J. Costa, and N. Camilleri, "Extracting small-signal model parameters of silicon MOSFET transistors," in IEEE MTT-S Int. Microwave Symp. Dig., San Diego, CA, May 1994, pp. 865-868.

[8] J. Wood and D. E. Root, "Bias-dependent linear scalable millimeterwave FET model," IEEE Trans. Microwave Theory Tech., vol. 48, no. 12, pp. 2352-2360, Dec. 2000.

[9] J. Wood, D. Lamey, D. C. M. Guyonnet, D. Bridges, N. Monsauret, and P. H. Aaen, "An extrinsic component parameter extraction method for high power RF LDMOS transistors," in IEEE MTT-S Int. Microwave Symp. Dig., Atlanta, GA, June 2008, accepted for publication.

[10] C. Fager, J. C. Pedro, N. B. de Carvalho, and H. Zirath, "Prediction of IMD in LDMOS transistor amplifiers using a new large-signal model," IEEE Trans. Microwave Theory Tech., vol. 50, no. 12, pp. 2834-42, Dec. 2002.

[11] D. Bridges, J. Wood, M. Guyonnet, and P. H. Aaen, "A nonlinear electrothermal model for high power RF LDMOS transistors," in IEEE MTTS Int. Microwave Symp. Dig., Atlanta, GA, June 2008, accepted for publication.

[12] W. R. Curtice, J. A. Plá, D. Bridges, T. Liang, and E. E. Shumate, "A new dynamic electro-thermal nonlinear model for silicon RF LDMOS FETs," in IEEE MTT-S Int. Microwave Symp. Dig., Anaheim, CA, June 1999, pp. 419-422.

[13] D. E. Root, "Charge modeling and conservation laws," in Asia-Pacific Microwave Conference Workshop WS2, 'Modeling and characterization of Microwave devices and packages', Sydney, Australia, June 1999.

[14] J. Xu, D. Gunyan, M. Iwamoto, A. Cognata, and D. E. Root, "Measurement-based non-quasi-static large-signal FET model using artificial neural networks," in IEEE MTT-S Int. Microwave Symp. Dig., San Francisco, CA, June 2006, pp. 469-472.

[15] G. Cybenko, "Approximation by superpostion of sigmodial functions," Mathematics of control, signals and systems, no. 2, pp. 303-314, 1989.

[16] S. Haykin, Neural Networks: a Comprehensive Foundation, 2nd ed. Upper Saddle River, NJ: Prentice Hall, 1995.

[17] C. M. Bishop, Neural Networks for Pattern Recognition. New York, NY: Oxford University Press, 1995.

[18] D. L. Chester, "Why two hidden layers are better than one," in Proc. Int. Joint Conf. Neural Networks, Washington, DC, Jan. 1990, pp. 265-268.

[19] L. Prechelt, "Automatic early stopping using cross validation:quantifying the criteria," Neural Networks, vol. 11, no. 4, pp. 761-767, 1998.

[20] P. C. Canfield, S. C. F. Lam, and D. J. Allstot, "Modeling of frequency and temperature effects in GaAs MESFETs," IEEE J. Solid State Circuits, vol. 25, no. 1, pp. 299-306, Feb. 1990.

[21] J.-M. Collantes, P. Bouysse, and R. Quere, "Characterising and modeling thermal behaviour of radio-frequency power LDMOS transistors," Electron. Lett., vol. 34, no. 14, pp. 1428-30, July 1998. 


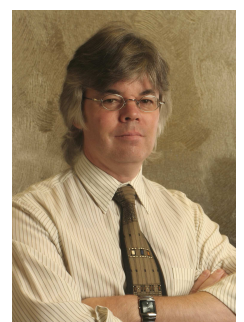

John Wood (M'87, SM'03, F'07) Received B.Sc. and $\mathrm{Ph} . \mathrm{D}$. degrees in Electrical and Electronic Engineering from the University of Leeds, UK, in 1976 and 1980 , respectively. He is a Senior Member of the Technical Staff, responsible for RF CAD \& Modeling in the RF Division of Freescale Semiconductor, Inc, Tempe, AZ, USA. From 1997-2005 he worked in the Microwave Technology Center of Agilent Technologies (then Hewlett Packard) in Santa Rosa, CA, USA. His areas of expertise include the development of compact device models and nonlinear behavioral models for RF power transistors and ICs. He is a Fellow of the IEEE, and a member of the Microwave Theory and Techniques, and Electron Devices Societies.

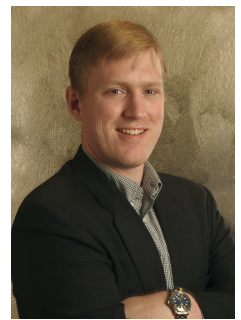

Peter H. Aaen (S'93, M'97) received the B.A.Sc. degree in Engineering Science and the M.A.Sc. degree in Electrical Engineering, both from the University of Toronto, Toronto, ON., Canada, and the $\mathrm{Ph} . \mathrm{D}$. degree in Electrical Engineering from Arizona State University, Tempe, AZ., USA, in 1995, 1997, and 2005, respectively. He is the Manager of the RF Modeling team of the RF Division of Freescale Semiconductor, Inc, Tempe, AZ, USA. His areas of interest include the development and validation of microwave transistor models, passive component modeling, and the electromagnetic simulation of complex packaged environments. He has co-authored Modeling and Characterization of $R F$ and Microwave Power FETs (Cambridge University Press, 2007), and has authored or co-authored of over a dozen papers.

Daren Bridges received the B.S. degree in electrical engineering from the University of Utah in 1992 and the M.S. degree in electrical engineering from the University of Texas at Dallas in 1996. In 1992, he joined the RF/Microwave Group at Texas Instruments and specialized in linear MESFET and HEMT modeling for MMIC devices. In 1997, he joined the Modeling team of the RF Division of Motorola Inc.s Semiconductor Products Sector (Freescale Semiconductor Inc.) His areas of expertise include RF high power nonlinear model development and implementation within harmonic balance simulators and the building and testing of active component model libraries. $\mathrm{He}$ is author or co-author on several papers, articles and workshops in the areas of RF and microwave device modeling and simulation.

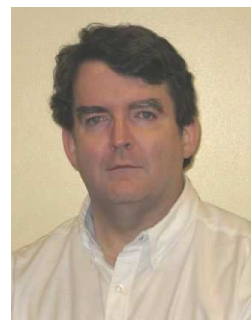

Dan Lamey received his M.S. Physics degree from the University of Minnesota. After joining Motorola's Semiconductor Products Sector in 1983 he worked in the Logic Division as a product analyst of TTL and ECL technologies, where he was device engineer in 1990, initially assigned to the technology introduction of Mixed-mode/RF BiCMOS. This lead to his subsequent involvement in LDMOS and GCMOS technology development. Since 2001 he has been a member of the RF Division of Freescale Semiconductor Inc., where he has been responsible for RF LDMOS integrated circuit CAD, as well as electromagnetic simulation and compact modeling of on-wafer passive devices.
Michael Guyonnet received his Ph.D. degree in microwaves \& RF from the University of Limoges, France, in 2005. While at the University of Limoges his work focused on the development of non linear electrothermal modeling techniques for LDMOS RF power transistors. In 2004 he joined Freescale Semiconductor Inc., as a senior modeling engineer and his work now concentrates on improving transistor linearity and advanced Doherty designs.

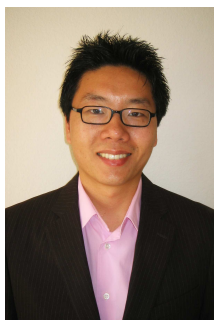

Daniel S. Chan (M'06) received the B.A.Sc. degree in electrical engineering from the University of Toronto, Toronto, Canada, in 2003. In 2004, he joined Qualcomm's CDMA Technologies division, San Diego, California, as an applications engineer. Since 2006, he has been a member of Freescale's RF Division, Tempe, Arizona, as a device modeling engineer where he has been responsible for RF LDMOS CAD and the development of passive component models.

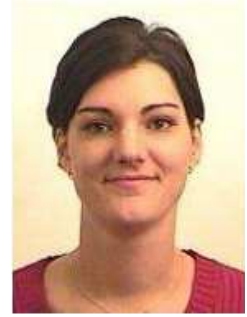

Nelsy Monsauret received a Ph.D. degree in microwaves \& RF from the University of Limoges, France, in 2001. Between 1997 and 2001, she worked on multi-band RF power amplifiers design in the wireless mobile group of Freescale Semiconductor (formerly Motorola Semiconductor), Toulouse, France. She joined the base-station power amplifier division of Freescale Semiconductor in 2001, as CAD and Design support. Since 2007, she has been working as RF modeling engineer in charge of developing transistor models. 